\title{
Friedrich Soennecken, Iserlohn-Dröschede (Sauerland) 1848 - Bonn 1919
}

Portrait d'un collectionneur

\section{Marie-Laure Ingelaere}

\section{OpenEdition}

Journals

Édition électronique

URL : https://journals.openedition.org/rbnu/3518

DOI : $10.4000 /$ rbnu. 3518

ISSN : 2679-6104

\section{Éditeur}

Bibliothèque nationale et universitaire de Strasbourg

Édition imprimée

Date de publication : 1 mai 2011

Pagination : 100-103

ISSN : 2109-2761

\section{Référence électronique}

Marie-Laure Ingelaere, « Friedrich Soennecken, Iserlohn-Dröschede (Sauerland) 1848 - Bonn 1919 », La Revue de la BNU [En ligne], 3 | 2011, mis en ligne le 01 mai 2011, consulté le 08 août 2021. URL : http:// journals.openedition.org/rbnu/3518; DOI : https://doi.org/10.4000/rbnu.3518

\section{(ब) $(\Theta \odot$}

La Revue de la BNU est mise à disposition selon les termes de la Licence Creative Commons Attribution - Pas d'Utilisation Commerciale - Partage dans les Mêmes Conditions 4.0 International. 


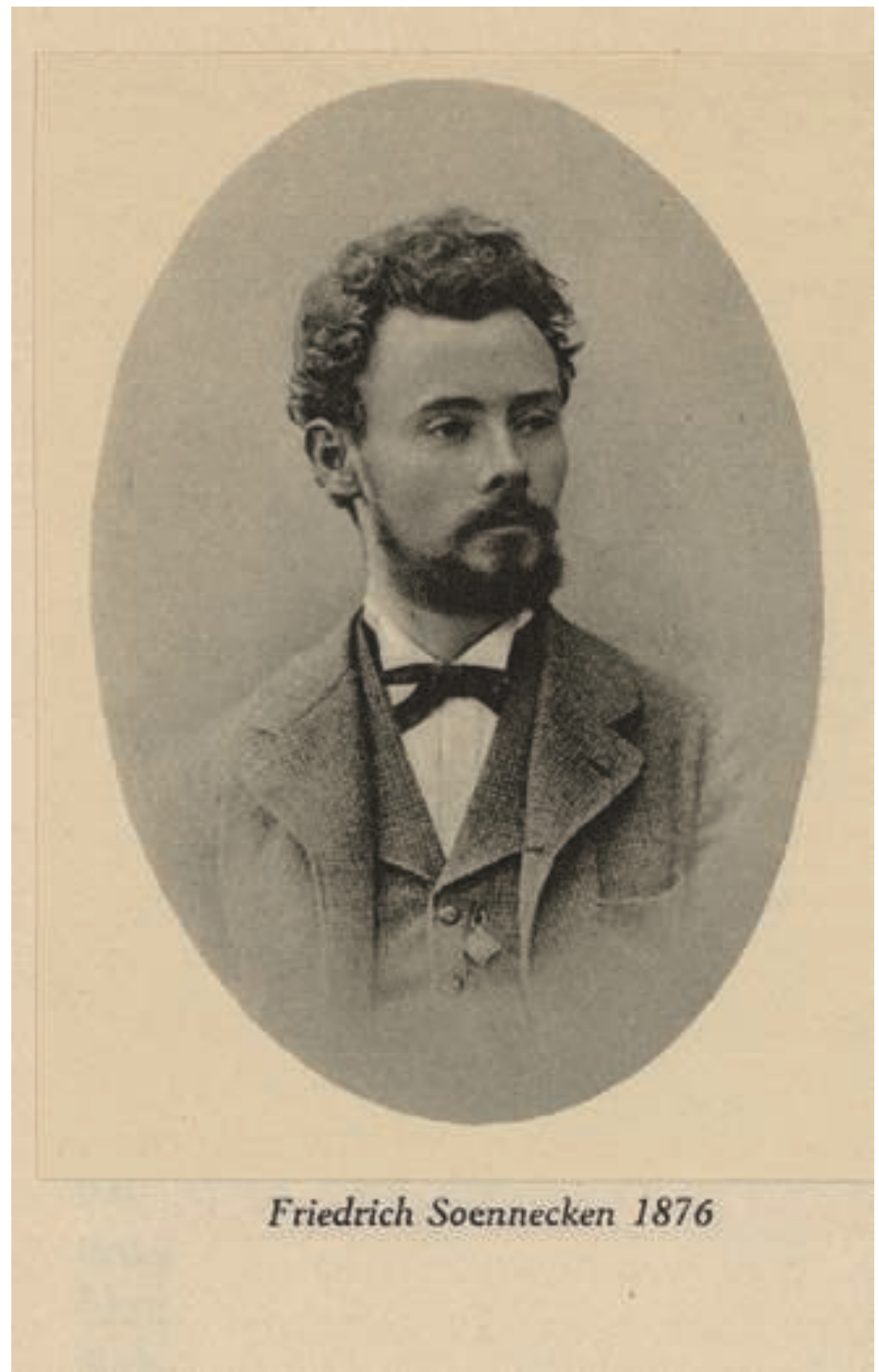

Portrait de Soennecken jeune, issu de l'ouvrage Friedrich Soennecken : ein Bahnbrecher in Schrift- und Schulfragen : Sonderdruck...

Essen : G. D. Baedeker, 1928 (coll. BNU) 


\section{Portrait d'un collectionneur : Friedrich Soennecken}

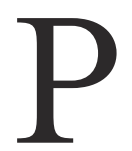

our les Allemands, le nom de Soennecken évoque l'univers du bureau. C'est en effet en 1875 à Bonn que Friedrich Soennecken fonda son entreprise. Cet industriel inventif mit toute son ingéniosité au service d'une incessante recherche dans ses domaines d'excellence : le matériel en relation avec le travail de bureau - sa devise : un bureau toujours en ordre - et l'écriture, base du travail dans les bureaux à cette époque. C'est lui qui inventa en 1886 un objet devenu banal, le classeur à anneaux, et pour en compléter l'usage, la perforatrice à feuilles. Il acquit une réputation incontestée dans ce domaine. Une lettre de Nietzsche envoyée à un ami en 1888 en témoigne : il a enfin trouvé un papier et une plume de qualité sortis de la manufacture Soennecken de Bonn !

Lorsqu'il était apprenti, Friedrich Soennecken avait commencé sa formation par l'initiation à l'écriture et à la calligraphie. Bien plus tard, devenu imprimeur, il fut confronté à la question de la normalisation des polices de caractères typographiques, un enjeu économique qui était la conséquence du développement industriel au XIX ${ }^{\mathrm{e}}$ siècle. De plus, la question du type d'écriture se posait dans une Allemagne qui était la seule en Europe à pratiquer l'écriture gothique alors que, dans les pays voisins comme la France, on avait adopté depuis longtemps la " ronde ". En Allemagne, où la question de la normalisation des caractères d'imprimerie suscitait débats et controverses au plus haut niveau, entre partisans et adversaires de la " Fraktur " (l'écriture gothique) et de "l'Antiqua " ${ }^{1}$, Friedrich Soennecken prit très tôt le parti de l'adoption de la " ronde " qu'il trouvait plus facile à écrire et plus élégante.
C'est ainsi qu'il publia dès 1877 une méthode d'apprentissage de l'écriture, en livrets, traduite en plusieurs langues. En 1881, il écrivit un ouvrage qui fit date sur la nécessité de la réforme de l'écriture allemande ${ }^{2}$.

Tous ses travaux reposaient sur la solide documentation qu'il s'était constituée avec rigueur, le "Soennecken Schriftmuseum ", à la fois musée et bibliothèque. D'un côté, il avait rassemblé une importante collection d'objets en relation avec l'écriture (encriers, écritoires, porte-plumes...) ${ }^{3}$. Et parallèlement, il avait constitué une bibliothèque de travail dont le noyau se trouva être l'acquisition, en 1878, des ouvrages ayant appartenu au calligraphe de la cour de Prusse à Berlin, Ernst Schütze. Il compléta cette belle collection par de nombreux achats au cours des années suivantes pour atteindre près de 2000 références. Cette bibliothèque fut mise en vente en 1968 par la maison Leitz à qui les héritiers de Soennecken avaient vendu leur entreprise. Elle a finalement été acquise par la Bibliothèque nationale et universitaire de Strasbourg.

Même si une partie des ouvrages a disparu du fait des guerres - le catalogue de la vente comporte 882 numéros - la collection est précieuse : les grandes bibliothèques possèdent bien sûr des ouvrages en relation avec la calligraphie et l'écriture, mais il n'existe cependant que peu de bibliothèques particulières dont les ouvrages n'ont pas été dispersés lors de leur vente et qui, de ce fait, ont gardé leur cohérence intellectuelle.

L'écriture telle qu'elle apparaît au travers de quatre siècles de production d'ouvrages de tous les genres, des manuscrits en passant par les incunables 
jusqu'aux ouvrages du début du $\mathrm{XX}^{\mathrm{e}}$ siècle, telle est l'ampleur chronologique de ce fonds ! Quant à l'origine des titres, elle englobe de vastes horizons géographiques, ce qui rend cet ensemble encore plus intéressant et instructif à l'heure de l'ouverture des frontières : ils proviennent en effet de toutes les contrées de l'Europe.

Un article paru en 1928 permet de connaître la composition primitive de la bibliothèque constituée par Friedrich Soennecken. Selon l'analyse très détaillée d'A. Legrün ${ }^{5}$, elle rassemblait en majorité des ouvrages pour l'enseignement de l'écriture qui provenaient des principaux pays européens : Allemagne, Autriche, Suisse, France, Belgique, Hollande, Angleterre, Espagne et Portugal. Il s'y trouvait les noms des plus grands maîtres de l'art de la calligraphie de ces pays. Elle comprenait des manuscrits, des incunables, des éditions produites entre le $\mathrm{XVI}^{\mathrm{e}}$ et le début du $\mathrm{XX}^{\mathrm{e}}$ siècle, avec une prédominance des ouvrages imprimés aux XVIII ${ }^{e}$ et $\mathrm{XIX}^{\mathrm{e}}$ siècles. Les formats étaient très divers, du plus modeste abécédaire, parfois sous forme de petits étuis de fiches modèles, au plus somptueux grand folio illustré de planches soigneusement gravées. Toutes les techniques graphiques y étaient représentées (bois, cuivre, acier, lithographies...).

Le catalogue de la vente montre que le fonds a gardé tout son intérêt même s'il présente l'ensemble selon un classement différent de celui adopté primitivement par Friedrich Soennecken, ce qui a entraîné parfois la dispersion en plusieurs endroits d'ouvrages dont la thématique était semblable ${ }^{6}$. Le catalogue distingue plusieurs parties. La première rassemble 71 manuscrits rares et précieux qui ont rejoint la Réserve de la BNU de Strasbourg. Les 241 ouvrages sur le thème de l'écriture et de l'histoire de l'écriture ont été rassemblés ; viennent ensuite les éditions anciennes et exemples concernant l'art de l'écriture (au nombre de 16). Comme dans l'état originel de la bibliothèque, les ouvrages d'enseignement de l'écriture, les méthodes et les abécédaires sont les plus nombreux (431 références). L'histoire de l'écriture, de l'imprimerie et du livre n'a pas été négligée puisqu'elle est représentée par un peu plus d'une centaine d'ouvrages.

$\mathrm{Au}$ travers du fonds Soennecken, c'est l'Europe de l'écriture qui nous est perceptible et qui s'offre à la découverte. Chaque nation a joué un rôle dans le développement de l'écriture, patrimoine qui nous devient d'autant plus commun que son évolution est maintenant aussi conditionnée par un nouveau support, le support numérique et un nouveau média de dimension universelle, Internet. Quelle évolution va connaître l'écriture alors que le clavier prend de plus en plus souvent la place de la plume?

Mais feuilleter les ouvrages dont est fait le fonds Soennecken ne présente pas seulement un intérêt intellectuel : la calligraphie n'est-elle pas l'art de dessiner et d'orner les caractères de la plus belle manière ? Lorsqu'on ouvre ces livres dont la variété des formats intrigue déjà, on en oublie le temps qui passe : comment détacher son regard de ces curieuses ornementations toutes différentes les unes des autres ? Sous nos yeux, les caractères se métamorphosent en véritables chefs-d'œuvre artistiques ! L'esthétique nous incite aussi à ouvrir les pages de ces magnifiques et rares ouvrages pour découvrir avec ravissement et surprise la beauté unique des lettres qui se transforment parfois en arabesques légères et inattendues !

\section{Marie-Laure Ingelaere}

\section{Notes}

1 - Voir Silvia Hartmann, Fraktur oder Antiqua. Der Schriftstreit von 1881 bis 1941, Frankfurt a. M., P. Lang, 1998 (Theorie und Vermittlung der Sprache; 28)

2 - Friedrich Soennecken, Das deutsche Schriftwesen und die Notwendigkeit seiner Reform, Bonn, Fr. Soennecken, 1881. Voir aussi, du même, Fraktur und Antiqua im ersten Unterricht? Vortrag... mit 50 Abbildungen, 1913

3 - En partie dispersée par les guerres et en partie conservée actuellement au musée de Cloppenburg. Cf. Elfriede Heinemeyer, Schreibgarnituren aus der Sammlung Kommerzienrat F. Soennecken, in Materialien zur Volkskultur nordwestliches Niedersachsen, 17, 1991

4 - Voir par exemple : David P. Becker, The Practice of Letters. The Hofer collection of writing manuals 1514-1800, Cambridge, Mass., Harvard College Library, 1997

5 - A. Legrün, Das Schriftmuseum F. Soennecken in Bonn (nebst einem Streifzug durch die Geschichte der Federhaltung), in Archiv für Schreibund Buchwesen, 2, 1928, p. 136-149. Cet article analyse la composition primitive de la bibliothèque avant les disparitions d'ouvrages causées par la guerre.

6 - Georges Fréchet, Schreibmeisterbücher aus der Bibliothèque nationale et universitaire de Strasbourg, in : Helmut Ottenjann (éd.), Handschrift, Schreib-Werke, Schrift und Schreibkultur im Wandel in regionalen Beispielen des 18. bis 20. Jahrhunderts, in Materialien zur Volkskultur nordwestliches Niedersachsen, Heft 16, Cloppenburg, 1991. Karl-Heinz Ziessow, Schrift als Handwerk : Schreibmeisterbücher des 16. bis 19. Jahrhunderts aus der Sammlung von Friedrich Soennecken, in : ibidem, p. 127-156 


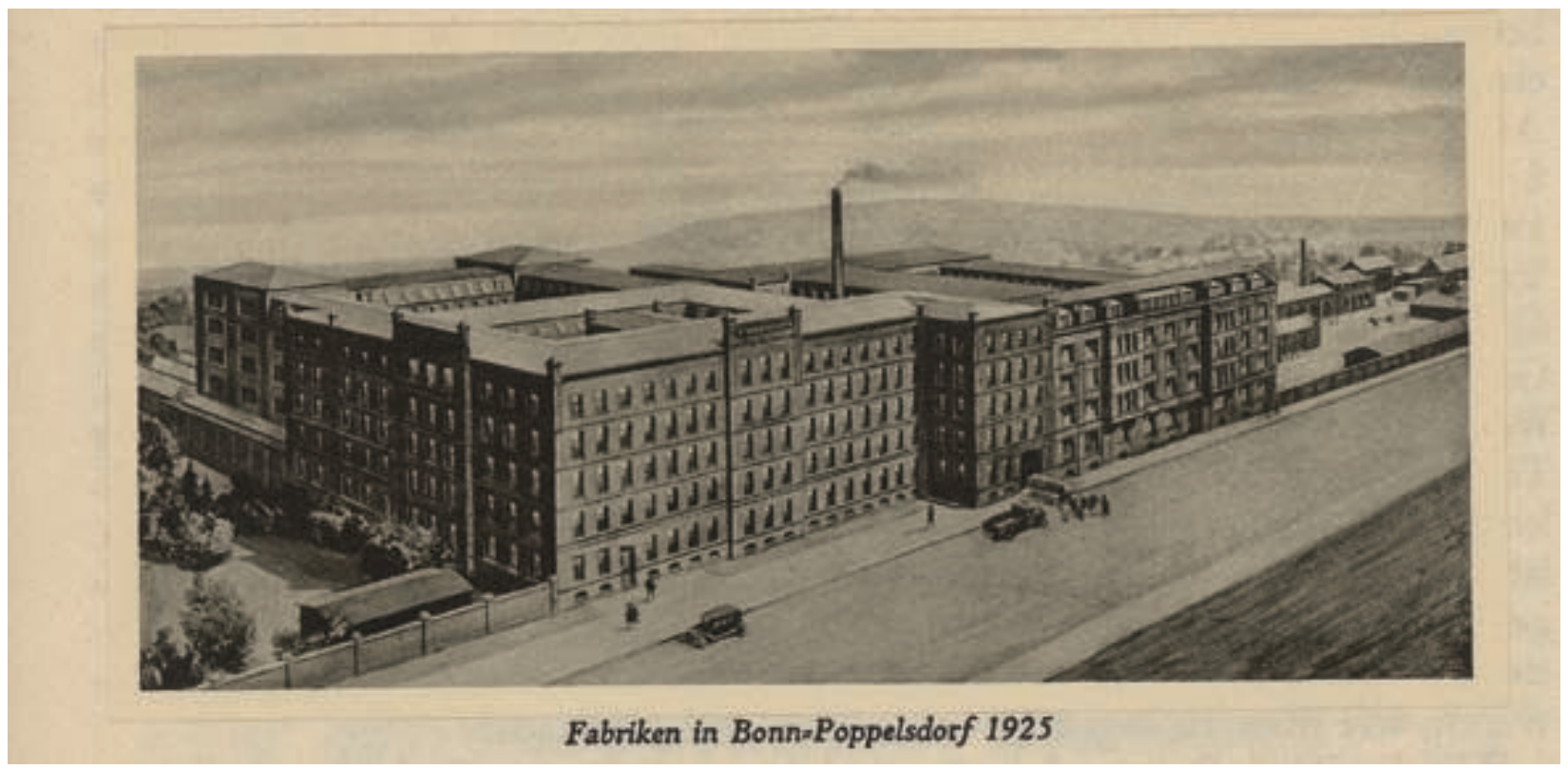

L'usine Soennecken dans la banlieue de Bonn (coll. BNU) 